\title{
Desenvolvimento da broca-das-cucurbitáceas em diferentes tipos de substratos alimentares
}

\author{
Dirceu Pratissolii ${ }^{1}$; Ricardo Antônio Polanczyk ${ }^{1}$; Anderson Mathias Holtz² ${ }^{2}$ Juliéder G Cocheto $^{1}$; Tiago \\ Tamanhoni'; André M Milanez ${ }^{1}$ \\ ${ }^{1}$ UFES, CCA, Depto. Produção Vegetal, C. postal 16, 29500-000 Alegre-ES; ${ }^{2}$ Escola Agrotécnica Federal de Colatina, C. postal 256, \\ 29709-910 Colatina-ES; pratissoli@cca.ufes.br; aholtz@insecta.ufv.br
}

\section{RESUMO}

Aspectos biológicos de Diaphania nitidalis foram estudados sob dietas natural e artificial em condições de laboratório, à temperatura de $25 \pm 1^{\circ} \mathrm{C}$, umidade relativa de $70 \pm 10 \%$ e fotofase de 14 horas. Como dieta natural foi utilizada abóbora 'Jacaré', pepino 'Japonês' e abobrinha 'Caserta' e, como artificial, a dieta utilizada em criações de Diatraea saccharalis, broca da cana-de-açúcar. As lagartas de $D$. nitidalis foram inoculadas nos diferentes tipos de substratos e criadas por todo o ciclo. Ocorreu diferença significativa entre a maioria dos parâmetros testados dentro dos tratamentos, sendo as dietas à base de abóbora e artificial as que proporcionaram maior potencial biótico para criação de $D$. nitidalis. Pela facilidade de aquisição dos ingredientes e manipulação dos insetos, a dieta artificial se torna mais eficaz para criação massal dessa espécie em laboratório.

\begin{abstract}
\section{Development of melonworm on different feeding substrates}

The aim of this research was to evaluate the biological behaviour of melonworm at different natural and artificial diet under laboratory conditions $\left(25^{\circ} \mathrm{C}, 70 \% \mathrm{RH}, 14 \mathrm{~h}\right.$ photofase $)$. Squash 'Jacaré', cucumber 'Japonês' and zucchini 'Caserta' were used as natural substrates compared to na artificial diet developed for the sugarcane borer (Diatraea saccharalis). The caterpillars were inoculated in each substrate and reared for a full cycle. The biological parameters were different among treatments. An artificial diet and squash cv. Jacaré substrate showed higher performance for the multiplication of $D$. nitidalis. The artificial diet is recommended because it is easy to obtain the ingredients and nultiply this insect under laboratory conditions.
\end{abstract}

Palavras-chave: Diaphania nitidalis, insecta, biologia de insetos.

Keywords: Diaphania nitidalis, insecta, insect biology.

(Recebido para publicação em 13 de dezembro de 2006; aceito em 22 de novembro de 2007)

$\mathrm{A}^{\mathrm{s}}$ plantas olerícolas pertencentes à família das cucurbitáceas constituem alimento de grande importância, principalmente em áreas tropicais, tanto pelo aspecto econômico como pelo nutricional, já que seus frutos possuem altos teores de açúcares, vitaminas e amido (Saturnino et al., 1982). Entre as espécies cultivadas no Brasil, abóboras, melancias, melões e pepinos exigem grandes investimentos, devido à freqüiente ocorrência de pragas e doenças (Filgueira, 2003).

Entre as pragas que atacam essa cultura, as brocas-das-cucurbitáceas, Diaphania hyalinata e D. nitidalis (Lepidoptera: Crambidae) são consideradas pragas-chave, causando injúrias que podem chegar, em algumas situações, até $100 \%$ de perdas na produção (Picanço \& Marquini, 1999; Picanço et al., 2000).

Para contornar tais problemas, tradicionalmente o controle de Diaphania spp. somente é realizado através da utilização de inseticidas químicos (Pratissoli, 2002). Contudo, o uso intensivo desses inseticidas pode provocar o ressurgimento da praga-alvo, bem como o aparecimento de novas pragas, já que a maioria desses produtos possui alto nível de ação biológica e persistência no ambiente, prejudicando assim a saúde do consumidor e dos profissionais envolvidos nos processos de produção (Brito et al., 2004). Os mesmos autores argumentam que deve-se levar em consideração a importância dos insetos polinizadores, bem como o resíduo deixado por esses produtos, visto que a tendência do mercado mundial é procurar por alimentos onde não foram utilizados produtos que possam comprometer a saúde das pessoas como também o meio ambiente.

Objetivando a busca de medidas alternativas ao controle químico, pesquisas relacionadas à criação massal de insetos em laboratório possibilitam o conhecimento mais detalhado sobre aspectos envolvidos no comportamento e biologia, oferecendo suporte para programas relacionados ao Manejo Integrado de Pragas (MIP) (Parra, 2000).

O sucesso dos programas de MIP depende, em grande parte, da habilidade das criações em laboratório desenvolvidas em dietas que ofereçam todas as condições nutricionais para os insetos completarem seu desenvolvimento. Desta forma, a quantidade e a qualidade do alimento consumido na fase larval podem interferir em parâmetros biológicos, tais como taxa de crescimento, tempo de desenvolvimento, peso do corpo, sobrevivência, fecundidade, longevidade, movimentação e capacidade de competição entre adultos (Parra, 1991). O objetivo deste trabalho foi avaliar as características biológicas de $D$. nitidalis em quatro substratos alimentares, com a finalidade de selecionar o melhor alimento, para sua criação massal em laboratório.

\section{MATERIAL E MÉTODOS}

O experimento foi realizado em laboratório, em sala climatizada com $25 \pm 1^{\circ} \mathrm{C}$, umidade relativa de $70 \pm 10 \%$ e fotofase de 14 horas. Inicialmente, lagartas de $D$. nitidalis foram coletadas em plantios comerciais de abóbora. Após a coleta, as lagartas foram levadas para laboratório e mantidas sobre o mesmo substrato alimentar proveniente do campo. Folhas de abóboras foram colocadas em recipientes do tipo "Tupperware" (30 x 20 x $10 \mathrm{~cm})$ e, sobre as folhas, foram colocadas 100 lagartas de D. nitidalis. Diariamente eram realizados os procedimentos de limpeza dos recipientes e troca das folhas. 
Tabela 1. Características biológicas de Diaphania nitidalis criada sobre diferentes substratos alimentares em laboratório $\left(25 \pm 1^{\circ} \mathrm{C} ; 70 \pm 10 \%\right.$ UR; fotofase de $14 \mathrm{~h}$ (Biological aspects of Diaphania nitidalis under different feeding substrates $\left(25 \pm 1^{\circ} \mathrm{C} ; 70 \pm 10 \% \mathrm{RH} ; 14 \mathrm{~h}\right.$ photofase). Alegre (ES), UFES, 2005.

\begin{tabular}{lcccc}
\hline Parâmetro & Pepino 'Japonês' & Abóbora 'Jacaré' & Abobrinha 'Caserta' & Dieta artificial \\
\hline Fase larval (dias) & $13,04 \pm 0,03 \mathrm{AB}$ & $13,36 \pm 0,12 \mathrm{~A}$ & $9,86 \pm 0,21 \mathrm{C}$ & $12,56 \pm 0,06 \mathrm{~B}$ \\
Viabilidade larval (\%) & $83,00 \pm 0,06 \mathrm{AB}$ & $83,00 \pm 0,00 \mathrm{AB}$ & $78,00 \pm 0,06 \mathrm{~B}$ & $92,00 \pm 0,03 \mathrm{~A}$ \\
Fase de pré-pupa (dias) & $1,57 \pm 0,10 \mathrm{AB}$ & $1,37 \pm 0,01 \mathrm{~B}$ & $1,69 \pm 0,07 \mathrm{AB}$ & $1,77 \pm 0,03 \mathrm{~A}$ \\
Fase de pupa (dias) & $9,29 \pm 0,09 \mathrm{~B}$ & $9,52 \pm 0,14 \mathrm{AB}$ & $9,80 \pm 0,05 \mathrm{~A}$ & $9,60 \pm 0,11 \mathrm{AB}$ \\
Viabilidade de pupa (\%) & $75,00 \pm 0,02 \mathrm{~A}$ & $84,00 \pm 0,00 \mathrm{~A}$ & $81,00 \pm 0,06 \mathrm{~A}$ & $84,00 \pm 0,04 \mathrm{~A}$ \\
Razão sexual & $0,51 \pm 0,01 \mathrm{~A}$ & $0,47 \pm 0,12 \mathrm{~A}$ & $0,54 \pm 0,01 \mathrm{~A}$ & $0,56 \pm 0,01 \mathrm{~A}$ \\
Peso de pupas macho (g) & $0,10 \pm 0,00 \mathrm{~B}$ & $0,12 \pm 0,00 \mathrm{~A}$ & $0,10 \pm 0,00 \mathrm{~B}$ & $0,13 \pm 0,00 \mathrm{~A}$ \\
Peso de pupas fêmea (g) & $0,10 \pm 0,00 \mathrm{C}$ & $0,12 \pm 0,00 \mathrm{AB}$ & $0,11 \pm 0,00 \mathrm{~B}$ & $0,13 \pm 0,00 \mathrm{~A}$ \\
Longevidade dos adultos (dias) & $7,81 \pm 0,07 \mathrm{~A}$ & $6,22 \pm 0,20 \mathrm{~B}$ & $7,75 \pm 0,23 \mathrm{~A}$ & $7,13 \pm 0,41 \mathrm{AB}$ \\
Número médio de ovos & $250,36 \pm 24,60 \mathrm{BC}$ & $363,63 \pm 11,85 \mathrm{AB}$ & $205,75 \pm 6,80 \mathrm{C}$ & $386,75 \pm 15,11 \mathrm{~A}$ \\
Período de incubação (dias) & $4,00 \pm 0,00 \mathrm{~A}$ & $4,00 \pm 0,00 \mathrm{~A}$ & $4,00 \pm 0,00 \mathrm{~A}$ & $4,00 \pm 0,00 \mathrm{~A}$ \\
Viabilidade dos ovos (\%) & $80,73 \pm 0,16 \mathrm{~B}$ & $87,85 \pm 0,56 \mathrm{~A}$ & $79,65 \pm 0,37 \mathrm{~B}$ & $89,09 \pm 0,54 \mathrm{~A}$ \\
\hline
\end{tabular}

Médias seguidas pela mesma letra, nas linhas, não diferem entre si, pelo teste de Tukey a $5 \%$ de probabilidade (means followed by the same letter in the row did not differ from each other, Tukey's test $\mathrm{p}<0.05)$.

Com a mudança para a fase de pupa, essas foram retiradas e transferidas para recipientes de acrílico transparente (11,5 x 11,5 x $3,5 \mathrm{~cm}$ ), contendo em seu interior um chumaço de algodão umedecido. Após 24 horas, as pupas foram sexadas, separadas e transferidas para novos recipientes.

Após a emergência, os adultos foram transferidos para gaiolas confeccionadas com pedaços de tubos de PVC (15 cm de diâmetro) de $20 \mathrm{~cm}$ de comprimento. A face superior do tubo foi fechada com tecido do tipo filó e a face inferior fechada com isopor recoberto com papel filtro. No interior da gaiola e sobre o isopor foi colocado um frasco contendo um chumaço de algodão embebido em uma solução de mel a 10\%, para alimentação dos adultos. Sobre o filó, na face superior do tubo, foi colocado um disco de papel de filtro, do mesmo diâmetro do tubo, para ser realizada a postura. Como estimulante para a postura, fatias circulares de pepino foram colocadas sobre o papel de filtro. Diariamente, os discos de papel foram retirados e armazenados em recipientes de acrílico transparente até a eclosão das larvas.

Após a primeira geração em laboratório, as lagartas de $D$. nitidalis foram divididas em grupos e criadas sobre os substratos alimentares a serem testados por duas gerações (em condições laboratoriais, conforme descrito anteriormente) a fim de se evitar o condicio- namento pré-imaginal. Os substratos utilizados para o experimento foram abóbora 'Jacaré', pepino 'Japonês', abobrinha 'Caserta' e a dieta artificial proposta por Hensley \& Hammond (1968) para criação de Diatraea saccharalis, broca da cana-de-açúcar.

Para cada dieta a ser testada, foram separadas 200 lagartas de D. nitidalis provenientes da mesma dieta. Nas dietas naturais, as lagartas foram acondicionadas individualmente em "gerbox" (6 $\mathrm{cm}$ de diâmetro x $2 \mathrm{~cm}$ de altura) com o devido substrato. A cada dois dias foram realizados os processos de limpeza dos "gerbox" e substituição do alimento. Na dieta artificial foram preparados $10 \mathrm{~mL}$ da mistura e depositados em tubos de ensaio $(8,5 \times 2,4 \mathrm{~cm})$. Foi colocada uma lagarta de $D$. nitidalis por tubo de dieta, devidamente esterilizado e tamponado com algodão hidrofóbico.

Após o desenvolvimento larval, as pupas obtidas em cada dieta foram retiradas e acondicionadas em caixas plásticas (25 x $10 \mathrm{~cm}$ de altura), permanecendo nestes recipientes por 24 horas. Posteriormente, essas pupas foram pesadas em balança analítica e sexadas, com auxilio de um microscópio estereoscópico. A unidade experimental utilizada nesta fase foi de cinco indivíduos, perfazendo um total de 40 repetições por substrato alimentar.

Em seqüência à emergência, os adultos foram separados em casais, perfazendo um total de 50 casais para cada substrato alimentar testado. Estes casais foram individualizados em gaiolas confeccionadas por tubos de PVC, papel toalha, isopor, solução de mel (alimentação) e rodelas de pepino (conforme descrição anterior). Cada repetição foi composta por uma gaiola e um casal de D. nitidalis, totalizando 50 repetições por substrato alimentar. Diariamente foi realizada a coleta dos ovos, retirando o disco de papel que foi oferecido no dia anterior. O disco de papel contendo os ovos foi armazenado em caixas plásticas ( 25 x $10 \mathrm{~cm}$ de altura) até a eclosão.

Foram avaliados a duração e viabilidade larval, duração de pré-pupa e pupa, viabilidade pupal, razão sexual, peso de pupas (machos e fêmeas), longevidade dos adultos (machos e fêmeas), numero médio de ovos por fêmeas, período de incubação e viabilidade dos ovos. Os resultados obtidos foram submetidos à análise de variância, e as médias comparadas pelo teste de Tukey, ao nível de 5\% de probabilidade.

\section{RESULTADOS E DISCUSSÃO}

A duração da fase larval variou de 9,86 a 13,36 dias, sendo as dietas artificial e à base de abóbora e de pepino as que apresentaram o maior tempo de desenvolvimento $(12,56,13,36$ e 13,04 dias, respectivamente), diferindo $(\mathrm{p}<0,05)$ do substrato abobrinha 'Caserta', que apresentou 9,86 dias de desenvolvimento (Tabela 1), indicando haver influência do alimento sobre essa 
fase no desenvolvimento de $D$. nitidalis . Smith (1911) encontrou valores médios de 14 dias para a fase larval deste inseto. Porém, Fulton (1947), utilizando folhas tenras e posteriormente pedaços de abóbora, observou 9 a 10 dias de período larval, resultados estes semelhantes ao da dieta natural de abobrinha 'Caserta'. Quando se comparam substratos alimentares, aquele que proporciona menor duração do ciclo é o melhor para o desenvolvimento biológico dos indivíduos envolvidos (Santos et al., 2000). Os alimentos mais adequados propiciam menor duração das fases de desenvolvimento e maior sobrevivência (Parra, 2000; Nava \& Parra, 2005).

O maior percentual de sobrevivência larval foi obtido na dieta a base de pepino 'Japonês', abóbora 'Jacaré' e na dieta artificial, com valores de 83,83 e $92 \%$, respectivamente (Tabela 1 ). As diferenças ocorridas entre as dietas provavelmente sejam devido às variações nos teores nutricionais dos alimentos testados, pois se trata de uma praga polífaga (Ali et al., 1990; Bemtancourt et al., 2004). Estes resultados diferiram $(\mathrm{p}<0,05)$ da dieta abobrinha 'Caserta' que apresentou $78 \%$ de sobrevivência (Tabela 1). Contudo, a dieta abobrinha também é considerada adequada, segundo Singh (1983) e Bavaresco et al. (2004), que indicam que é aceitável um mínimo de $75 \%$ de sobrevivência para que uma dieta seja considerada adequada para o desenvolvimento de um inseto.

Na fase de pré-pupa, a duração média foi de 1,37; 1,57; 1,69 e 1,77 dias para as dietas abóbora, pepino, abobrinha e dieta artificial, respectivamente (Tabela 1), sendo que, entre os tratamentos, apenas a dieta abóbora foi significativamente menor $(\mathrm{p}<0,05)$ que a dieta artificial.

Para a duração da fase de pupa, ocorreu diferença $(\mathrm{p}<0,05)$ somente entre as dietas pepino (9,29 dias) e abobrinha (9,8 dias) (Tabela 1). No entanto, os tipos de dietas testadas não influenciaram a viabilidade pupal, ficando seus valores entre 75 e $84 \%$ (Tabela 1). Estes resultados indicam que o condicionamento alimentar proporcionado pelas dietas em questão não interferiram no desenvolvimento desta praga.
A razão sexual foi semelhante entre os tratamentos, com valores entre 0,47 a 0,56 (Tabela 1). Mendes \& Berti Filho (1981) encontraram valores de 0,46 para $D$. nitidalis criadas em melão e de 0,49 em dieta artificial.

O tipo de alimento fornecido às lagartas de $D$. nitidalis influenciou significativamente $(\mathrm{p}<0,05)$ o peso de pupas, tanto para machos quanto para fêmeas, ficando os valores entre 0,10 e $0,13 \mathrm{~g}$ para ambos. Os maiores valores foram encontrados na dieta à base de abóbora e na dieta artificial, com 0,12 e 0,13 g, respectivamente (Tabela 1 ). Estes resultados indicam uma preferência alimentar ou até mesmo uma adequação nutricional a estes alimentos, pois de acordo com Santos \& Boiça Junior (2001) e Santos et al. (2005), um menor peso de pupa indicaria a não-preferência alimentar. Segundo Santos (2000), o alimento é um fator condicionante na biologia dos insetos, podendo ser seletivo quanto ao vigor dos indivíduos.

No parâmetro longevidade dos adultos, observa-se que houve influência quanto ao tipo de substrato alimentar utilizado na criação de $D$. nitidalis. Os adultos provenientes da dieta abóbora apresentaram longevidade de 6,22 dias, enquanto os adultos provenientes das dietas abobrinha e pepino apresentaram 7,75 e 7,81 dias, respectivamente (Tabela 1). De acordo com Santos et al. (2000), estas características são significativamente influenciadas por uma adequação de dietas alimentares. Estes resultados indicam que uma menor duração da fase de lagarta proporciona indivíduos com melhor desenvolvimento biológico (Santos et al., 2000).

O número médio de ovos por fêmeas nos diferentes substratos alimentares variou entre 205,75 a 386,75 ovos (Tabela 1). A maior capacidade de oviposição foi apresentada pelas fêmeas que foram alimentadas com a dieta artificial (386,75 ovos) e com a dieta abóbora $(363,63$ ovos), onde a primeira delas diferiu significativamente dos demais substratos testados (Tabela 1). Johansson (1964) e Santos et al. (2005) relatam que alguns parâmetros reprodutivos como a produção de ovos pode ser influenciada por diferenças físicas ou químicas do alimento ou pela quantidade ingerida. Estes resultados indicam que a dieta artificial e a dieta abóbora atendem melhor às exigências nutricionais para este inseto que, segundo Parra (2000) são os nutrientes essenciais (aminoácidos, vitaminas e sais minerais) e não essenciais (carboidratos, lipídios e esteróis). Além disso, estes devem estar adequadamente balanceados, principalmente na relação proteína $\mathrm{x}$ carboidratos.

Os diferentes tipos de substratos alimentares não influenciaram no período de incubação dos ovos de $D$. nitidalis, os quais levaram um período médio de quatro dias de incubação. Em trabalhos realizados com outras espécies de herbívoros, o período de incubação também se mostrou invariável quando comparado a diferentes tipos de substratos alimentares (Foerster \& Dionísio, 1989; Machado et al., 1985; Santos et al., 2005).

No parâmetro de viabilidade de ovos, observa-se que os substratos à base de abobrinha e pepino foram os que apresentaram a menor viabilidade. Nos outros dois tipos de dieta, observou-se uma taxa acima de $87 \%$ (Tabela 1). Essa diferença pode estar relacionada à influência do alimento na fase larval sobre características reprodutivas dos adultos (Santos et al., 2005).

Dentre os parâmetros biológicos analisados, alguns se mostraram invariáveis com o tipo de alimento utilizado. Contudo, a dieta à base de abóbora 'Jacaré' e a dieta artificial proporcionaram maior potencial biótico para criação de $D$. nitidalis em laboratório. Entretanto, pela facilidade de aquisição dos ingredientes e manipulação dos insetos, a dieta artificial se torna mais eficaz para criação massal dessa espécie em laboratório.

\section{REFERÊNCIAS}

ALI A; LUTTRELL RG; SCHNEIDER JC. 1990. Effects of temperature and larval diet on development of the fall armyworm (Lepidoptera: Noctuidae). Annals of Entomological Society of America 83: 725-733.

BAVARESCO A; GARCIA MS; GRÜTZMACHER AD; RINGENBERG R; FORESTI J. 2004. Adequação de uma dieta artificial para a criação de Spodoptera cosmioides (Walk.) (Lepidoptera: Noctuidae) em laboratório. Neotropical Entomology 33: 155-161. 
BEMTANCOURT CM; SCATONI IB GONZALEZ A; FRANCO J. 2004. Biology of Bonagota cranaodes (Meyrick) (Lepidoptera: Tortricidae) on seven natural foods. Neotropical Entomology 33: 299-306.

BRITO GG; COSTA EC; MAZIERO H; BRITO AB; DÖRR FA. 2004. Preferência da brocadas-cucurbitáceas Diaphania nitidalis Cramer, 1782 (Lepidoptera: Pyralidae) por cultivares de pepineiro em ambiente protegido. Ciência Rural 34: 577-579.

FILGUEIRA FAR. 2003. Novo manual de olericultura. Viçosa: UFV. 412p.

FOERSTER LA; DIONÍSIO ALM. 1989. Necessidades térmicas de Spodoptera eridania (Cramer, 1782) (Lepidoptera: Noctuidae) em Bragatinga (Mimosa scabrella BENTHAM) (leguminosae). Anais da Sociedade Entomológica do Brasil 18: 145-154.

FULTON BB. 1947. Biology and control of the picklewrm. North carolina Agricultural Experiment station: West Raleigh, Tecnical Bulletin 85. 27p.

HENSLEY SD; HAMMOND AH. 1968 Laboratory techniques for rearing the sugar cane borer on an artificial diet. J. Econ. Entomol 61: 1742-1743.

JOHANSSON AS. 1964. Feeding and nutrition in reproductive processes in insects. Symp. $R$. Entomol 2: 43-55.

LARA FM. 1991. Princípios de resistência de plantas aos insetos. São Paulo: Ícones. 336p.
MACHADO VLL; GIANNOTTI E; OLIVEIRA RM. 1985. Aspecto biológico de Spodoptera frugiperda (Smith, 1797) (Lepidoptera: Noctuidae) em couve (Brassica oleracea L. var. acephala). Anais da Sociedade Entomológica do Brasil 14: 123-130.

MENDES ACB; BERTI FILHO E. 1981. Biologia da broca das cucurbitáceas Diaphania nitidalis (Cramer, 1781) (Lepidoptera: Pyralidae). Anais da Sociedade Entomológica do Brasil 10: 141-146.

NAVA DE; PARRA JRP. 2005. Biologia de Stenoma cantenifer Walsingham (Lepidoptera: Elachistidae) em dieta natural e artificial e estabelecimento de um sistema de criação. Neotropical Entomology 34: 751-759.

PARRA JRP. 2000. Técnicas de criação de insetos para programas de controle biológico. Piracicaba: ESALQ/FEALQ. 138p.

PARRA JRP. 1991. Consumo e utilização de alimentos por insetos. In: PANIZZI AR; PARRA JRP (eds). Ecologia nutricional de insetos e suas implicações no manejo de pragas. São Paulo: Manole. p.9-65.

PICANÇO M; MARQUINI F. 1999. Manejo integrado de pragas de hortaliças em ambiente protegido. Informe Agropecuário 20: 126-133.

PICANÇO M; GUSMÃO MR; GALVAN TL. 2000. Manejo integrado de pragas de hortaliças. In: ZAMBOLIM L (eds). Manejo integrado: doenças, pragas e plantas daninhas. Viçosa: UFV. p.275-324.
PRATISSOLI D. 2002. Manejo de pragas em hortaliças com ênfase em controle biológico. In: Memórias Sociedad Colombiana de Entomologia SOCOLEN. Monteria, Colômbia. p.5-10.

SANTOS KB; MENEGUIM AM; NEVES PMOJ. 2005. Biologia de Spodoptera eridania (Cramer) (Lepidoptera: Noctuidae) em diferentes hospedeiros. Neotropical Entomology. 34: 903-910.

SANTOS TM; BOIÇA JUNIOR AL. 2001. Resistência de genótipos de algodoeiro (Gossypium hirsutum L.) a Alabama argillacea (Hübner) (Lepidoptera: Noctuidae). Neotropical Entomology 30: 297-303.

SANTOS GP; ZANUNCIO TV; ZANUNCIO JC. 2000. Desenvolvimento de Thyrinteina arnobia Stoll (Lepidoptera: Geometridae) em folhas de Eucalyptus urophylla e Psidium guajava. Anais da Sociedade Entomológica do Brasil 29: 13-22.

SATURNINO HM; PAIVA BM; GONTIJO VPM; FERNANDES DPL; VIEIRA GS. 1982. Cucurbitáceas: aspectos estatísticos. Informe Agropecuário 8: 3-20.

SINGH P. 1983. A general purpose laboratory diet mixture for rearing insects. Insects Science Applied 4: 357-362.

SMITH RI. 1911. Two important cantaloup pests. North Carolina Agricultural Experiment Station: West Raleigh, Technical Bulletin 214. 42p. 